\title{
Predator persistence through variability of resource productivity in Tritrophic systems
}

\section{Soudijn, Floor Helena; de Roos, Andre M.}

\section{Published in:}

American Naturalist

Link to article, DOI:

$10.1086 / 694119$

Publication date:

2017

Document Version

Publisher's PDF, also known as Version of record

Link back to DTU Orbit

Citation (APA):

Soudijn, F. H., \& de Roos, A. M. (2017). Predator persistence through variability of resource productivity in Tritrophic systems. American Naturalist, 190(6), 844-853. https://doi.org/10.1086/694119

\section{General rights}

Copyright and moral rights for the publications made accessible in the public portal are retained by the authors and/or other copyright owners and it is a condition of accessing publications that users recognise and abide by the legal requirements associated with these rights.

- Users may download and print one copy of any publication from the public portal for the purpose of private study or research.

- You may not further distribute the material or use it for any profit-making activity or commercial gain

- You may freely distribute the URL identifying the publication in the public portal 
Note

\title{
Predator Persistence through Variability of Resource Productivity in Tritrophic Systems
}

\author{
Floor H. Soudijn ${ }^{1,2, \star}$ and André M. de $\operatorname{Roos}^{1}$ \\ 1. Institute for Biodiversity and Ecosystem Dynamics, University of Amsterdam, Amsterdam, The Netherlands; \\ 2. Centre for Ocean Life, National Institute for Aquatic Resources, Technical University of Denmark, Charlottenlund, Denmark \\ Submitted October 19, 2016; Accepted May 26, 2017; Electronically published August 31, 2017 \\ Online enhancements: appendix with two parts, source code, header file, R scripts.
}

\begin{abstract}
A BSTRACT: The trophic structure of species communities depends on the energy transfer between trophic levels. Primary productivity varies strongly through time, challenging the persistence of species at higher trophic levels. Yet resource variability has mostly been studied in systems with only one or two trophic levels. We test the effect of variability in resource productivity in a tritrophic model system including a resource, a size-structured consumer, and a size-specific predator. The model complies with fundamental principles of mass conservation and the body-size dependence of individual-level energetics and predatorprey interactions. Surprisingly, we find that resource variability may promote predator persistence. The positive effect of variability on the predator arises through periods with starvation mortality of juvenile prey, which reduces the intraspecific competition in the prey population. With increasing variability in productivity and starvation mortality in the juvenile prey, the prey availability increases in the size range preferred by the predator. The positive effect of prey mortality on the trophic transfer efficiency depends on the biologically realistic consideration of body size-dependent and food-dependent functions for growth and reproduction in our model. Our findings show that variability may promote the trophic transfer efficiency, indicating that environmental variability may sustain species at higher trophic levels in natural ecosystems.
\end{abstract}

Keywords: predator-prey dynamics, prey size structure, starvation mortality, variable productivity, predator persistence, tritrophic food chain.

\section{Introduction}

The transfer of energy between different trophic layers shapes the trophic structure of species communities. At the base of the trophic web, energy is generated by basal species or primary producers. This productivity varies substantially through time in natural systems. For example, the productivity of phytoplankton in marine and aquatic systems and of

\footnotetext{
* Corresponding author; e-mail: f.h.soudijn@uva.nl. ORCIDs: de Roos, http://orcid.org/0000-0002-6944-2048.
}

Am. Nat. 2017. Vol. 190, pp. 844-853. (C) 2017 by The University of Chicago 0003-0147/2017/19006-57339\$15.00. All rights reserved. This work is licensed under a Creative Commons Attribution 4.0 International License (CC BY 4.0), which permits reuse of the work with attribution.

DOI: $10.1086 / 694119$ plants in terrestrial systems shows characteristic seasonal patterns (e.g., Gebauer et al. 2002; Platt et al. 2003; Klausmeier and Litchman 2012). In addition, productivity may vary in response to large, irregular influxes of nutrients or rainfall (Ostfeld and Keesing 2000; Yang et al. 2010). These fluctuations in productivity permeate to species at higher trophic levels (Ostfeld and Keesing 2000; Platt et al. 2003; Willson and Womble 2006; Yang et al. 2010; Klausmeier and Litchman 2012). Fluctuations in resource productivity are therefore expected to shape ecological as well as evolutionary dynamics of many different species (Holt 2008; Nakazawa and Doi 2012; Sainmont et al. 2014).

The effect of resource variability on population and community dynamics has been tested in a range of population models (Holt 2008). In discrete-time, single-population models, variability in population growth rate can affect the average population growth rate both positively and negatively (Lewontin and Cohen 1969; Caswell 2001; Lawson et al. 2015). In continuous-time models, consumer abundance often decreases (Nisbet and Gurney 1976; Cushing 1986) but may also increase (Cushing 1986) with resource variability. Resource fluctuations are known to promote competitive coexistence of consumers (Armstrong and McGehee 1976; Sommer 1985; Hambäck 1998; Chesson 2000; Kuang and Chesson 2008) but may also reduce it (Holt 2008). In addition, periodic variation in resources may lead to resonance phenomena that either decrease (Nisbet and Gurney 1976) or increase (Nisbet and Gurney 1976; Hanski et al. 1993; Rinaldi et al. 1993) the tendency of systems to cycle and display chaotic dynamics. Intrinsically generated population cycles can, similar to resource fluctuations, promote competitive coexistence (Armstrong and McGehee 1976; Huisman and Weissing 1999; Chesson 2000), but large-amplitude oscillations also increase the risk of species extinctions as densities travel close to zero. In summary, resource variability can have a profound effect, whether positive or negative, on species persistence and coexistence. Yet to date the effect of resource variability has mostly been studied in systems with up to two trophic levels. 
In multitrophic systems, the consequences of resource variability are intimately linked to the trophic transfer of energy, which results from the feeding of consumers on resources. Such interactions occur between individuals and not between species (Clark et al. 2011). Yet classic population models generally make assumptions on the population level and fail to make the essential link between the individual and the population level (for an exception, see Yodzis and Innes 1992). Modeling the trophic transfer of energy in a multitrophic system requires a consistent accounting of the energy flow from individuals up to populations of different species (de Roos and Persson 2005; Persson et al. 2014). In contrast to classic population models, size-structured population models are based on assumptions at the individual level regarding the intake and use of energy (Metz and Diekmann 1986; de Roos and Persson 2002, 2013). These assumptions lead to body size-dependent and food-dependent functions for growth, reproduction, and mortality (Kooijman 2000). Size-structured population models furthermore allow for size-dependent, life stage-specific feeding interactions between predator and prey that are generally considered important for trophic energy transfer (Peters 1983; Ebenman and Persson 1988; Brose et al. 2006; Rudolf and Lafferty 2011). Using a size-structured approach therefore results in a consistent description of the flow of energy through the population and model system that complies with the fundamental principle of mass conservation, the size dependence of individual-level energetics, and the allometry of predator-prey interactions.

In this study, we analyze the effect of variability in resource productivity on trophic transfer efficiency using a model system with three trophic layers including an unstructured resource, a size-structured consumer, and a size-specific predator. Our results show that with increasing variability, the consumer population increasingly suffers from starvation mortality. This starvation mortality occurs whenever food assimilation is not sufficient to cover the energy requirements for somatic maintenance costs. As a result of the additional mortality, consumer biomass increases in certain size ranges, that is, the consumer size distribution changes. The predator benefits from this effect of variability in resource productivity; it is able to sustain a much higher harvesting pressure and is less prone to suffer from a catastrophic collapse with a fluctuating resource productivity than with a constant resource productivity.

\section{Model Description}

To study the effect of variable resource productivity on predator-consumer-resource dynamics, we use a model system with three trophic levels including an unstructured resource, a size-structured consumer, and an unstructured sizespecific predator. The consumer population is represented by a stage-structured biomass model described previously by de Roos et al. (2008b). Predator dynamics follow bioenergetic assumptions as first described by Yodzis and Innes (1992) and discussed in more detail in de Roos and Persson (2013, chap. 4). The model has been used extensively before (de Roos et al. 2008a; Ohlberger et al. 2011; de Roos and Persson 2013). A description of the model details and the computational techniques used here can be found in the appendix, available online. We summarized the equations in table A1 and the parameter values we used in table A2 (tables A1, A2 are available online).

Under equilibrium conditions, the stage-structured biomass model constitutes an exact approximation of a model accounting for a complete size distribution of juveniles between their size at birth and their size at maturation and a class of nongrowing adult individuals (de Roos et al. 2008b). The model follows the generic bioenergetic approach introduced by Yodzis and Innes (1992). Growth, reproduction, and mortality are assumed to be resource-dependent processes. Individuals can invest in growth or reproduction only if the energy obtained through resource ingestion exceeds the costs of maintenance. When the resource ingestion is not sufficient to cover the maintenance cost, no growth, maturation, and reproduction takes place, and starvation mortality occurs. Juvenile consumers invest all surplus energy in growth; adults do not grow and invest all surplus energy in reproduction. Following Yodzis and Innes (1992), all energetic processes (resource intake, maintenance, growth in body size, and reproduction) are assumed proportional to body size, while individuals are assumed to experience a size-independent per capita background mortality rate. The model can account for differences in mass-specific resource ingestion or per capita mortality rate between, but not within, the juvenile and the adult stage. In this article, we analyze a model parameterization in which adults have a higher mass-specific resource ingestion rate than juveniles while both are foraging on the same resource. The predator is assumed to forage on adult individuals only.

The difference from previous studies using the same model (de Roos et al. 2007; de Roos and Persson 2013) pertains to the resource productivity function. The resource follows semichemostat growth with a productivity term that is not constant but varies over time:

$$
G(R)=\rho R_{\max }\left(1+\psi \sin \left(2 \pi \frac{t}{Y}\right)\right)-\rho R
$$

The resource growth $G(R)$ thus follows a sinusoidal function with period $Y$. The amplitude of the sinusoidal function is controlled by $\psi$. For $\psi=0$ no fluctuations occur, and when equal to its maximum value, $\psi=1$, the amplitude of fluctuations in resource growth equals the average resource productivity $\rho R_{\max }$, the product of resource turnover rate $\rho$, 
and the average maximum resource density $R_{\max }$. Notice that different values of $\psi$ do not affect the time-averaged value of resource productivity.

We analyze the effect of variability in resource productivity on the model system by varying the amplitude $(\psi)$ and period $(Y)$ of the fluctuations in the resource growth rate. In addition, the effect of variability in resource productivity is tested on the persistence of the top predator in the model system in relation to predator mortality. Finally, we test the robustness of our results to "pink" stochastic daily variation in the productivity of the resource as opposed to regular periodic variability using methods previously described by de Roos et al. (2008a) and Vasseur (2007; see the appendix).

\section{Results}

In the absence of predators and with constant resource productivity, biomass in the juvenile stage of the consumer is much higher than that in the adult stage (fig. 1). For our default parameter values, juveniles have a lower mass-specific resource ingestion rate than adults. Therefore, juvenile individuals mature relatively slowly to the adult stage, while the production of juvenile biomass by adults is high. This results
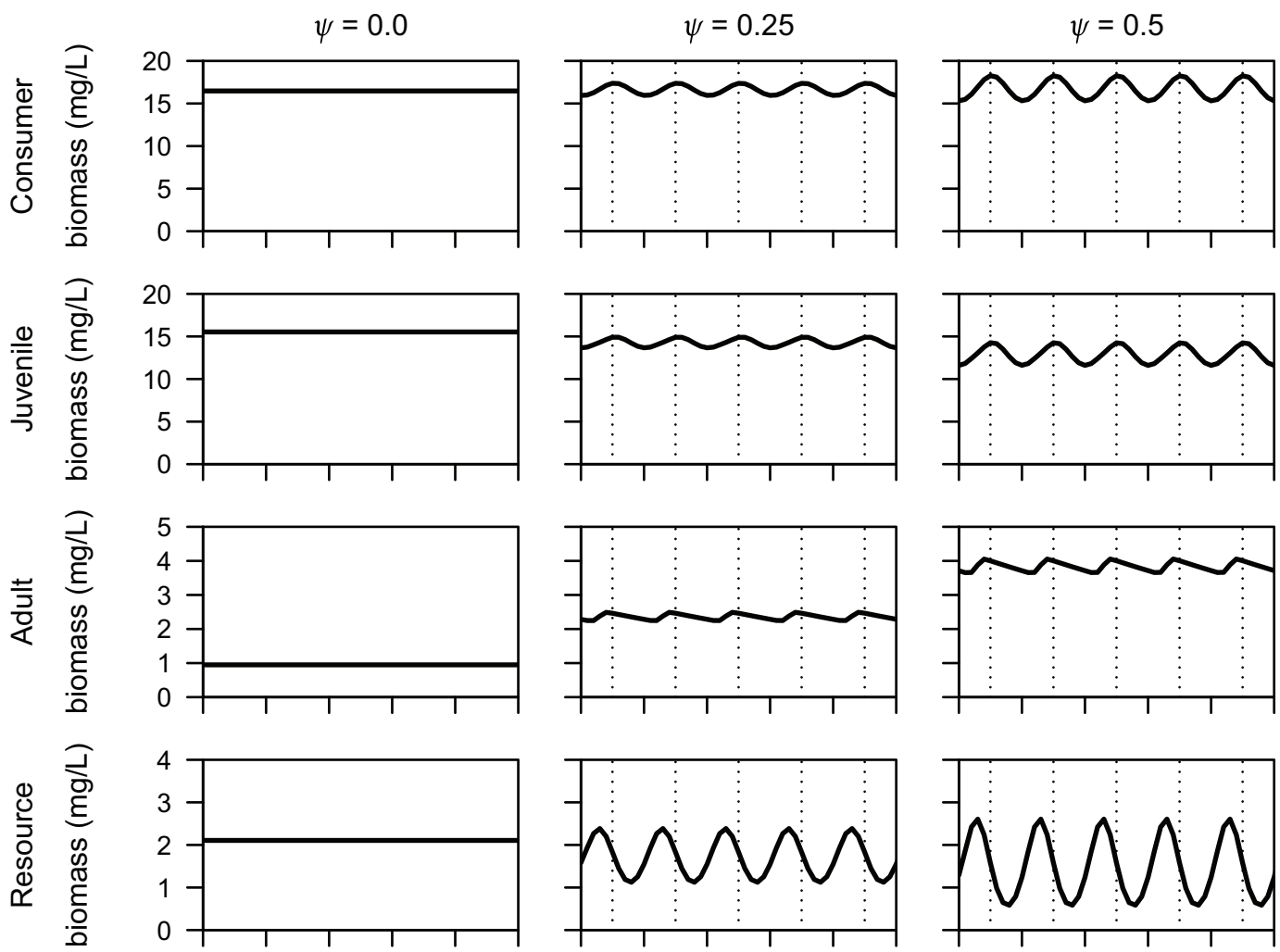

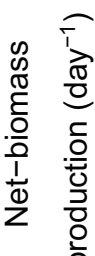
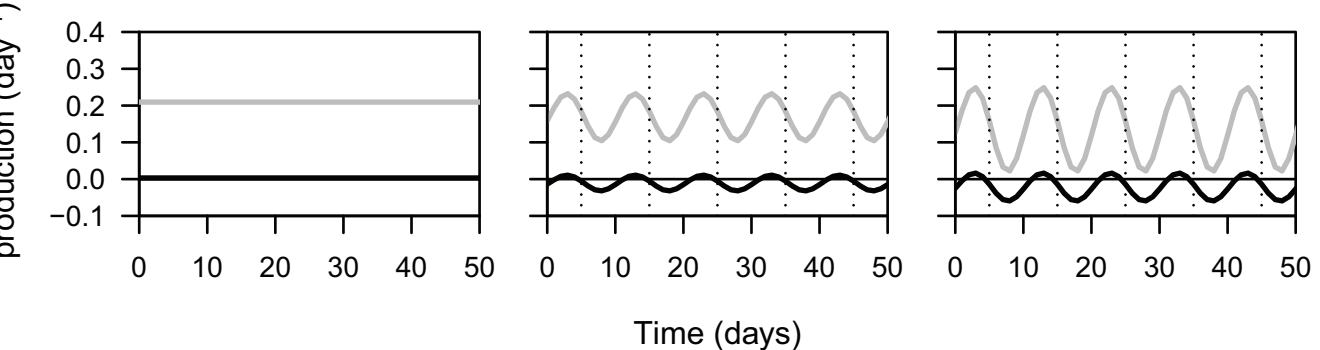

Time (days)

Figure 1: Resource-consumer dynamics for three values of $\psi(\psi=0.0, \psi=0.25, \psi=0.5)$ in the absence of predators. Plotted are the total consumer, juvenile, adult, and resource biomass and the mass-specific net biomass production rate of adults (gray lines) and juveniles (black lines) for default parameter values (table A2). Vertical dotted lines indicate the start of a period with a negative net biomass production rate of juveniles. 
in an accumulation of biomass in the juvenile stage. More generally, stage-specific differences in resource use always result in a skewed distribution of biomass between life stages or size classes in populations (de Roos et al. 2007).

Figure 1 shows that variability of resource productivity induces changes in the biomass distribution of the consumer. With increasing variability, the adult biomass increases while the juvenile biomass decreases. The variability in resource productivity causes distinct fluctuations in the resource density for values of $\psi>0$ (fig. 1). This results in periods of juvenile starvation mortality whenever the resource availability is too low to cover maintenance costs. When starving, growth and maturation of the juveniles halt, and juvenile biomass decreases due to starvation mortality. Adult biomass decreases due to background mortality only. When the consumer biomass has decreased sufficiently and the resource has increased sufficiently, the juvenile net biomass production becomes positive again. This results in an increase in juvenile biomass due to growth and an increase in adult biomass due to maturation of juveniles.

The starvation mortality in the juvenile stage relaxes intraspecific competition for food. The surviving juveniles mature faster, resulting in an increase in adult biomass on average (figs. 1, 2). Temporal food shortages result only in juvenile starvation and not in adult starvation because adults are more efficient foragers on the resource than juveniles. Note also that variability in resource productivity results in a suppression of the average resource density to slightly lower levels and a small increase in the average consumer biomass.

Variability in resource productivity promotes predator persistence (figs. 2, 3, A1; fig. A1 is available online). For a predator mortality rate that does not allow predator persistence with a constant resource productivity, a fluctuating resource productivity leads to conditions that allow for predator persistence (fig. $2 A, 2 B$ ). In addition, the average biomass of the predator increases with increasing variability in resource productivity. Resource variability benefits the adult-specialist predator through an increase in juvenile starvation mortality that results in an increase in adult consumer biomass. The increased food availability for the predator explains the predator persistence in the system at significantly higher predator mortality rates with varying resource productivity (fig. 3). Increasing the amplitude of the fluctuations in resource productivity results in a shift of the predator extinction boundary to higher predator mortality values (figs. 3, A1). Variability thus promotes the trophic energy transfer and the resilience of the predator to harvesting.

Stochastic variability in the resource productivity benefits the predator just like sinusoidal fluctuations do (fig. $2 B$ ). The coefficient of variation of the stochastic noise needs to reach relatively high values for the fluctuations to have an effect. With increasing stochastic fluctuations in productivity, star- vation mortality increases more slowly than with increasing sinusoidal fluctuations. For a very short or long period of the sinusoidal fluctuations in resource productivity, juvenile consumer starvation mortality decreases and the positive effect on the predator abundance decreases (fig. 2C). This can be explained by the response rates of both the resource and the consumer to the fluctuations. Fast fluctuations do not allow the time for strong changes in resource density, while slow fluctuations result in synchronous fluctuations of the resource and consumer. Yet the qualitative result remains that fluctuations in resource productivity allow for predator persistence where constant productivity does not, irrespective of the period of the resource fluctuations.

For a constant resource productivity (fig. $3 A$ ), alternative stable equilibria occur at low predator mortality values. Here, the system can exhibit two stable states, depending on initial conditions, in which the predator is either absent or present. When the predator is present at high density, predation mortality imposed by the predator on the adult consumers releases intraspecific competition, primarily among juveniles, and consequently increases maturation and the adult biomass. Yet once it has become extinct, the predator is unable to reinvade the system from low densities because its food availability is too low. The alternative stable states thus arise through a positive effect of the predator on its own food availability corresponding to an Allee effect. Since this Allee effect is not explicitly incorporated in the model equations, it is referred to as an emergent Allee effect (de Roos et al. 2003; de Roos and Persson 2013). The population bottleneck in the structured consumer thus results in predator collapses in response to additional predator mortality.

The predator is less prone to suffer from a mortalityinduced collapse at higher variability in resource productivity. With fluctuations in resource productivity, the range of mortality values over which the two stable patterns of (fluctuating) dynamics can occur becomes smaller (figs. $3 B, 3 C$, A1). Note that with fluctuating resource productivity stable and unstable dynamic patterns (or attractors) represent regular fluctuations with a period equal to the period of productivity fluctuations. For large-amplitude fluctuations the range of mortality values with alternative stable patterns of dynamics becomes marginally small (figs. $3 C$, A1). The fluctuations relax intraspecific competition through starvation mortality in the consumer, which increases adult biomass and thereby allows predator invasion where this would not be possible with constant resource productivity (fig. 3 ).

The fluctuations in resource productivity increase the persistence range of the predator with a region of stable types of dynamics with decreasing predator density, where the predator does not impose top-down control on the system (fig. 3). Instead, the dynamics are controlled "bottom-up," that is, the predator-consumer-resource coexistence dynamics are 

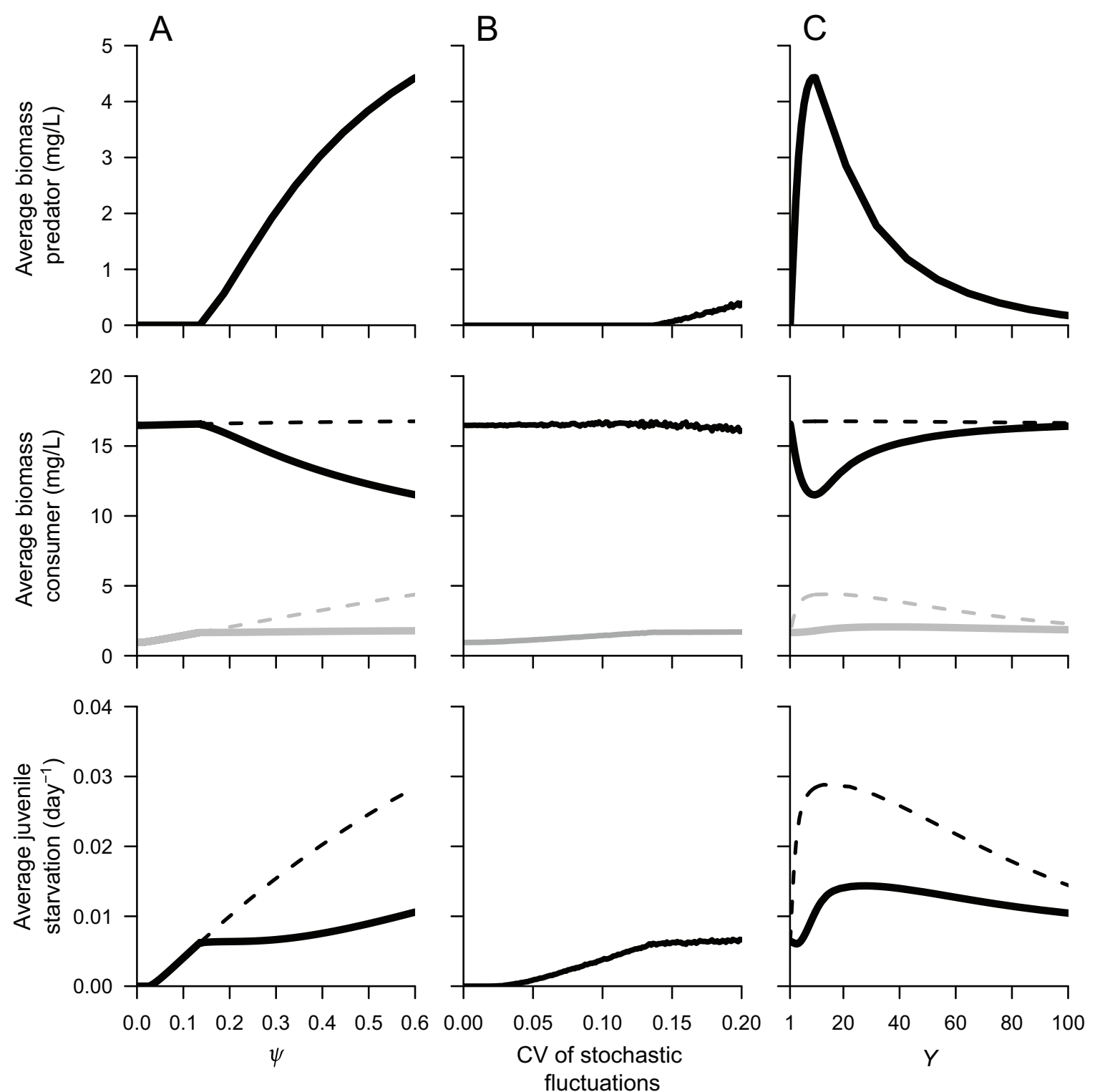

Figure 2: Average values of predator biomass (top panels), consumer biomass (middle panels, black solid lines), adult biomass (middle panels, gray lines), and the starvation mortality rate in the juvenile stage (bottom panels). Biomasses are plotted as a function of the relative amplitude of the sinusoid fluctuations of the resource productivity $\psi(A)$, the coefficient of variation $(\mathrm{CV})$ of stochastic fluctuations of the resource productivity $(B)$, and the period of the sinusoid fluctuations of the resource productivity $Y(C)$. In $A$ and $C$, average values for the consumer-resource system in the absence of the predator are also shown (dashed lines). We used default parameters (table A2) except for an additional predator mortality rate of $\mu_{P}=0.02$ (all three panels), a period of the sinusoid fluctuations of $Y=10(A)$, and an amplitude of $\psi=0.5(C)$. Note that the results in $A$ and $C$ follow from equilibrium computations, while the results in $B$ are derived using numerical simulations (for computational details, see the appendix).

characterized by a lower predator density, where resource competition among consumers leads to starvation mortality (figs. 3, A1, ivory region). Variability of resource productivity thus increases predator persistence and decreases the probability of predator collapse through a situation where bottomup regulation by the fluctuating resource abundance completely drives the dynamics.

\section{Discussion}

Previous studies have shown that resource variability can promote competitive coexistence and thereby increase the diversity of consumers (Armstrong and McGehee 1976; Sommer 1985; Chesson 2000). The trophic transfer efficiency may be stimulated through this mechanism (e.g., Leibold and Wil- 

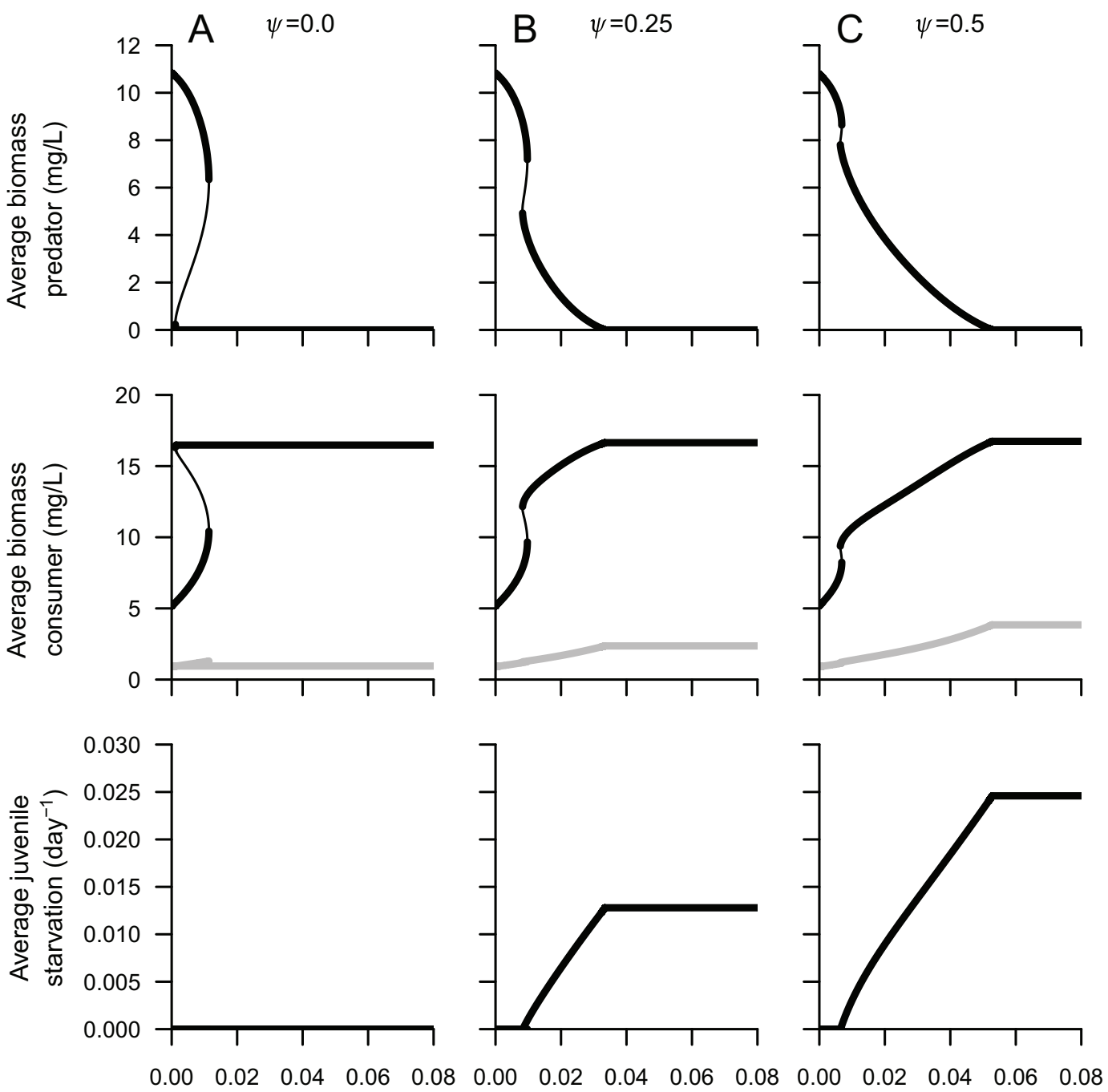

Additional predator mortality $\left(\right.$ day $\left.^{-1}\right)$

Figure 3: Average values of predator biomass (top panels), consumer biomass (middle panels, black), adult biomass (middle panels, gray), and juvenile consumer starvation (bottom panels) as a function of additional predator mortality for three values of $\psi(A, \psi=0.0 ; B, \psi=$ $0.25 ; C, \psi=0.5$ ). Thick and thin lines refer to stable and unstable dynamic patterns (attractors), respectively. Note that for $\psi=0$ the stable dynamic pattern corresponds to a stable equilibrium, while for $\psi>0$ the stable dynamic patterns correspond to stable regular cycles. Default parameter values (table A2) were used for calculations.

bur 1992). We show here that trophic transfer efficiency can be stimulated by variability directly in food chains with only a single consumer species. It has also been shown previously that resource variability may decrease or enhance system stability, as variability leads to resonance phenomena or interference with limit cycles that are intrinsic to the model system (Nisbet and Gurney 1976; Vasseur and Fox 2007). In contrast, our results show that variability can positively affect predator persistence compared with a situation without any fluctuations in the dynamics.

The effect of variable productivity has mainly been studied using models with only one or two trophic levels. On the other hand, the effect of variability in mortality has been studied using multitrophic systems, such as a nutrient-plantherbivore model (Thébault and Loreau 2005) and the diamond model (resource, two consumers, one predator; Vasseur and Fox 2007; Gouhier et al. 2010). These studies were mostly aimed at assessing community stability in the form of the amplitude of fluctuations in the populations and do not explicitly consider the effect of variability on trophic transfer efficiency. However, the metacommunity of diamond models described by Gouhier et al. (2010) shows a decreasing predator abundance with increasing variability in mortality due to an on average increased mortality in the consumers. Our 
results show an opposite effect: the increased starvation mortality in the juvenile stage of the consumer population, which results from variability in resource productivity, positively affects predator abundance.

The effect of resource variability on populations depends on the characteristics of individuals in different life stages in the population (Caswell 2001; Hastings 2014). The positive effect of variability on predator persistence that we show here depends critically on the assumption that periods with low food availability affect individuals in different life stages to a different extent. Many examples of temporal starvation mortality due to resource fluctuations have been reported for a range of species. The likelihood of surviving a seasonal resource low is often life stage dependent (Quinn 1988; Clutton-Brock et al. 1997; Sogard 1997). This size-dependent starvation mortality has been observed to affect population size distributions. It seems therefore reasonable to expect that the mechanism we theoretically reveal here for predator-prey coexistence also occurs in real ecosystems. Yet we know of no empirical or experimental study that identifies or was aimed at finding the coexistence mechanism that we present in this study. In experiments with protozoans feeding on bacteria, Friman and Laakso (2011) found that pulsed-resource dynamics resulted in a higher predator biomass than constantresource dynamics. They attributed this effect to a lower frequency of prey types with a defense mechanism against predation. In experiments with Tribolium, resource fluctuations were shown to release intraspecific competition and increase population biomass (Jillson 1980; Henson and Cushing 1997). In these experiments, however, the population biomass increase was attributed to a release of cannibalism rather than a release of intraspecific competition for food, as in the results we report here.

A positive effect of increased consumer mortality on the trophic transfer efficiency has also been found in studies that assume resource productivity to be constant (de Roos and Persson 2002; van Leeuwen et al. 2008; Schröder et al. 2014). This positive effect of mortality on the trophic transfer efficiency occurs as a result of so-called biomass overcompensation. An increase in size-specific biomass in response to mortality occurs because intraspecific competition, which normally inhibits the flow of biomass through the consumer population, is released. Similarly, the change in the consumer size distribution we report comes about through a release of the intraspecific competition in the consumer population by starvation mortality. It has been shown that biomass overcompensation becomes apparent only when both reproduction and growth are implemented as food-dependent processes and the fact that sustaining biomass requires energy for maintenance is explicitly accounted for (de Roos et al. 2007; Schröder et al. 2014). In our study, an overall decreased loss of energy to basic maintenance is also the ultimate reason for why trophic transfer efficiency can increase when starvation mortality of juvenile consumers occurs. In contrast to many of the previous theoretical studies that considered the effect of variability on population dynamics, we used a mechanistic size-structured modeling approach. Sizestructured models are based on individual-level assumptions regarding the acquisition and use of energy and trophic interactions (Metz and Diekmann 1986; de Roos and Persson 2002, 2013). The beneficial effect of variability in resource productivity on predator performance that we report underlines the importance of population size structure and the consideration of individual-level energetics for a mechanistic understanding of population and community dynamics.

Biomass overcompensation as a result of a shift in the population size distribution in response to increased mortality has been shown to occur in various experimental systems (Schröder et al. 2014), but it does not occur without a distinct bottleneck in either individual development or reproduction (Reichstein et al. 2015). Such a bottleneck translates into a difference in the flow of biomass between different size classes and an accumulation of biomass in a specific size class of the population. Our results show that resource variability potentially releases bottlenecks in development or reproduction. Such a release leads to a situation where biomass overcompensation in response to further increases in mortality due to other causes occurs to a lesser extent or not at all. This is illustrated by the disappearance of the emergent Allee effect with large variability in resource productivity in this study. The emergent Allee effect has been presented as an explanation for the lack of recovery following a population collapse of marine fish stocks, such as those that occurred in cod populations in the Northwest Atlantic and the Baltic Sea (van Leeuwen et al. 2008; Gårdmark et al. 2015). Our results indicate that in systems with seasonal fluctuations an emergent Allee effect can indeed occur, with alternative stable dynamics characterized by a high and a low predator density, respectively. Yet we also suggest that in highly variable environments, where seasonal starvation mortality is very high, the alternative types of dynamics are less likely to occur. This implies that other community-level effects attributed to biomass overcompensation (de Roos and Persson 2013), such as predator recovery after prey culling (Persson et al. 2007), predator-to-predator facilitation (Huss and Nilsson 2011), and pathogen-to-predator facilitation (Ohlberger et al. 2011), might also be less likely to occur in highly variable systems.

We focused on the effect of sinusoidal fluctuations in resource productivity, but figure $2 B$ shows that the trophic transfer of energy may also be stimulated by stochastic fluctuations in the resource productivity. Essential for the positive effect to occur is an increase in the periodic starvation mortality in the consumer population to a sufficiently high level. Moreover, the positive effect of variability in resource productivity on the trophic transfer efficiency is not depen- 
dent on the structure of the model for consumer dynamics. A model with a continuous consumer size structure, including growing adult consumers, shows qualitatively similar results (Soudijn 2016) to the stage-structured biomass model. The parameterization of this continuously size-structured model represents a piscivorous fish that preferentially forages on the small juvenile individuals of a planktivorous fish species (de Roos and Persson 2002). In the continuously size-structured model, small juvenile consumers have a competitive advantage over larger immature ones. On the other hand, in the stage-structured model used in this article the larger adult consumers are the superior competitors. The effect that we present in this article therefore occurs in a variety of community settings. For the increase in trophic transfer efficiency we present to occur, however, it is crucial that the predator forages on the part of the population that increases in response to mortality (the competitively superior size range of consumers).

In previous studies, both positive and negative effects of resource variability on species persistence and coexistence have been reported (Holt 2008). Yet the perception of the relationship between variability and system stability and diversity seems to be predominantly negative. This is illustrated by the fact that the amplitude of fluctuations in a model system is often used as a measure of community stability (e.g., Loreau and de Mazancourt 2013). Mechanisms such as differential reaction rates of species abundances to perturbations are therefore thought to neutralize the negative effects of resource variability in natural systems (Thébault and Loreau 2005; McCann and Rooney 2009; Loreau and de Mazancourt 2013). The negative reputation of variability seemingly arises from classic results, such as the negative effect of stochasticity on the population growth rate (Lewontin and Cohen 1969; Lawson et al. 2015) and the increased tendency of systems to fluctuate wildly with variability (Hanski et al. 1993; Rinaldi et al. 1993). Resource fluctuations have, however, been shown to promote competitive coexistence of species (Armstrong and McGehee 1976; Sommer 1985; Chesson 2000). Here we show that variability in resource productivity enables predator-prey coexistence. These findings indicate that the effects of environmental variability, especially of intermediate amplitude, can promote species coexistence and sustain species at higher trophic levels in natural ecosystems. They furthermore suggest that variable or seasonal environments may support communities with more trophic levels.

\section{Acknowledgments}

We thank the theoretical ecology group at the University of Amsterdam, A. van Leeuwen, C. Guill, and D. van Denderen for helpful discussions and comments on early versions of the manuscript. The research leading to these results has re- ceived funding from the University of Amsterdam, the Swedish Environmental Protection Agency, and the Swedish Board of Fisheries. The Centre for Ocean Life is supported by the Villum Foundation. F.H.S. is financially supported by the People Programme (Marie Curie Actions) of the European Union's Seventh Framework Programme (FP7/2007-2013) under Research Executive Agency grant agreement 609405 (COFUNDPostdocDTU). A.M.d.R. is supported by funding from the European Research Council (ERC) under the European Union's Seventh Framework Programme (FP/20072013)/ERC grant agreement 322814.

\section{Literature Cited}

Armstrong, R. A., and R. McGehee. 1976. Coexistence of species competing for shared resources. Theoretical Population Biology 9:317328.

Brose, U., R. J. Williams, and N. D. Martinez. 2006. Allometric scaling enhances stability in complex food webs. Ecology Letters 9:1228-1236.

Caswell, H. 2001. Matrix population models. 2nd ed. Sinauer, Sunderland, MA.

Chesson, P. 2000. Mechanisms of maintenance of species diversity. Annual Review of Ecology and Systematics 31:343-358.

Clark, J. S., D. M. Bell, M. H. Hersh, M. C. Kwit, E. Moran, C. Salk, A. Stine, D. Valle, and K. Zhu. 2011. Individual-scale variation, species-scale differences: inference needed to understand diversity. Ecology Letters 14:1273-1287.

Clutton-Brock, T. H., A. W. Illius, K. Wilson, B. T. Grenfell, A. D. C. MacColl, and S. D. Albon. 1997. Stability and instability in ungulate populations: an empirical analysis. American Naturalist 149: 195-219.

Cushing, J. M. 1986. Oscillatory population growth in periodic environments. Theoretical Population Biology 30:289-308.

de Roos, A. M., and L. Persson. 2002. Size-dependent life-history traits promote catastrophic collapses of top predators. Proceedings of the National Academv of Sciences of the USA 99:12907-12912.

- 2005. Unstructured population models: do population-level assumptions yield general theory? Pages 31-62 in B. Beisner and K. Cuddington, eds. Ecological paradigms lost: routes of theory change. Elsevier Academic, Burlington, MA.

- 2013. Population and community ecology of ontogenetic development. S. A. Levin and H. S. Horn, eds. Monographs in Population Biology 51. Princeton University Press, Princeton, NJ.

de Roos, A. M., L. Persson, and E. McCauley. 2003. The influence of size-dependent life-history traits on the structure and dynamics of populations and communities. Ecology Letters 6:473-487.

de Roos, A. M., T. Schellekens, T. van Kooten, and L. Persson. 2008a. Stage-specific predator species help each other to persist while competing for a single prey. Proceedings of the National Academv of Sciences of the USA 105:13930-13935.

de Roos, A. M., T. Schellekens, T. van Kooten, K. van de Wolfshaar, D. Claessen, and L. Persson. 2007. Food-dependent growth leads to overcompensation in stage-specific biomass when mortality increases: the influence of maturation versus reproduction regulation. American Naturalist 170:E59-E76.

2008b. Simplifying a physiologically structured population model to a stage-structured biomass model. Theoretical Population Biology 73:47-62. 
Ebenman, B., and L. Persson, eds. 1988. Size-structured populations. Springer, Berlin.

Friman, V.-P., and J. Laakso. 2011. Pulsed-resource dynamics constrain the evolution of predator-prey interactions. American Naturalist 177:334-345.

Gårdmark, A., M. Casini, M. Huss, A. van Leeuwen, J. Hjelm, L. Persson, and A. M. de Roos. 2015. Regime shifts in exploited marine food-webs: detecting mechanisms underlying alternative stable states using size-structured community dynamics theory. Philosophical Transactions of the Roval Society B 370:20130262.

Gebauer, R. L. E., S. Schwinning, and J. R. Ehleringer. 2002. Interspecific competition and resource pulse utilization in a cold desert community. Ecology 83:2602-2616.

Gouhier, T. C., F. Guichard, and A. Gonzalez. 2010. Synchrony and stability of food webs in metacommunities. American Naturalist 175:E16-E34.

Hambäck, P. A. 1998. Seasonality, optimal foraging, and prey coexistence. American Naturalist 152:881-895.

Hanski, I., P. Turchin, E. Korpimäki, and H. Henttonen. 1993. Population oscillations of boreal rodents: regulation by mustelid predators leads to chaos. Nature 364:232-235.

Hastings, A. 2014. Temporal scales of resource variability: effects on population dynamics of structured populations. Ecological Complexity 18:6-9.

Henson, S. M., and J. M. Cushing. 1997. The effect of periodic habitat fluctuations on a nonlinear insect population model. Journal of Mathematical Biology 36:201-226.

Holt, R. D. 2008. Theoretical perspectives on resource pulses. Ecology 89:671-681.

Huisman, J., and F. J. Weissing. 1999. Biodiversity of plankton by species oscillations and chaos. Nature 402:407-410.

Huss, M., and K. A. Nilsson. 2011. Experimental evidence for emergent facilitation: promoting the existence of an invertebrate predator by killing its prey. Journal of Animal Ecology 80:615-621.

Jillson, D. A. 1980. Insect populations respond to fluctuating environments. Nature 288:699-700.

Klausmeier, C. A., and E. Litchman. 2012. Successional dynamics in the seasonally forced diamond food web. American Naturalist 180: 1-16.

Kooijman, S. A. L. M. 2000. Dynamic energy and mass budgets in biological systems. 2nd ed. Cambridge University Press, Cambridge.

Kuang, J. J., and P. Chesson. 2008. Predation-competition interactions for seasonally recruiting species. American Naturalist 171:E119E133.

Lawson, C. R., Y. Vindenes, L. Bailey, and M. van de Pol. 2015. Environmental variation and population responses to global change. Ecology Letters 18:724-736.

Leibold, M. A., and H. M. Wilbur. 1992. Interactions between foodweb structure and nutrients on pond organisms. Nature 360:341-343.

Lewontin, R. C., and D. Cohen. 1969. On population growth in a randomly varying environment. Proceedings of the National Academy of Sciences of the USA 62:1056-1060.

Loreau, M., and C. de Mazancourt. 2013. Biodiversity and ecosystem stability: a synthesis of underlying mechanisms. Ecology Letters 16: 106-115.

McCann, K. S., and N. Rooney. 2009. The more food webs change, the more they stay the same. Philosophical Transactions of the Roval Societv B 364:1789-1801.

Metz, J. A. J., and O. Diekmann. 1986. The dynamics of physiologically structured populations. Vol. 68. Lecture Notes in Biomathematics. Springer, Berlin.
Nakazawa, T., and H. Doi. 2012. A perspective on match/mismatch of phenology in community contexts. Oikos 121:489-495.

Nisbet, R. M., and W. S. C. Gurney. 1976. Population dynamics in a periodically varying environment. $\underline{\text { Journal of Theoretical Biology }}$ 56:459-475.

Ohlberger, J., Ø. Langangen, E. Edeline, D. Claessen, I. Winfield, N. Stenseth, and L. Vollestad. 2011. Stage-specific biomass overcompensation by juveniles in response to increased adult mortality in a wild fish population. Ecology 92:2175-2182.

Ostfeld, R. S., and F. Keesing. 2000. Pulsed resources and community dynamics of consumers in terrestrial ecosystems. Trends in Ecology and Evolution 15:232-237.

Persson, L., P. Amundsen, A. M. de Roos, A. Klemetsen, R. Knudsen, and R. Primicerio. 2007. Culling prey promotes predator recoveryalternative states in a whole-lake experiment. Science 316:17431746.

Persson, L., and A. M. de Roos. 2013. Symmetry breaking in ecological systems through different energetic efficiencies of juveniles and adults. Ecology 94:1487-1498.

Persson, L., A. van Leeuwen, and A. M. de Roos. 2014. The ecological foundation for ecosystem-based management of fisheries: mechanistic linkages between the individual-, population-, and communitylevel dynamics. ICES Journal of Marine Science 71:2268-2280.

Peters, R. H. 1983. The ecological implications of body size. Cambridge University Press, Cambridge.

Platt, T., C. Fuentes-Yaco, and K. T. Frank. 2003. Spring algal bloom and larval fish survival. Nature 423:398-399.

Quinn, G. 1988. Ecology of the intertidal pulmonate limpet Siphonaria diemenensis Quoy et Gaimard. II. Reproductive patterns and energetics. Journal of Experimental Marine Biology and Ecology 117: 137-156.

Reichstein, B., L. Persson, and A. M. de Roos. 2015. Ontogenetic asymmetry modulates population biomass production and response to harvest. Nature Communications 6:6441.

Rinaldi, S., S. Muratori, and Y. Kuznetsov. 1993. Multiple attractors, catastrophes and chaos in seasonally perturbed predator-prey communities. Bulletin of Mathematical Biology 55:15-35.

Rudolf, V. H. W., and K. D. Lafferty. 2011. Stage structure alters how complexity affects stability of ecological networks. Ecology letters 14:75-79.

Sainmont, J., K. H. Andersen, Ø. Varpe, and A. W. Visser. 2014. Capital versus income breeding in a seasonal environment. American Naturalist 184:466-476.

Schröder, A., A. van Leeuwen, and T. C. Cameron. 2014. When less is more: positive population-level effects of mortality. Trends in Ecology and Evolution 29:614-624.

Sogard, S. M. 1997. Size selective mortality in the juvenile stages of teleost fishes: a review. Bulletin of Marine Science 60:11291157.

Sommer, U. 1985. Comparison between steady state and non-steady state competition: experiments with natural phytoplankton. Limnology and Oceanography 30:335-346.

Soudijn, F. H. 2016. Populations exposed to seasonal variability: from individual-level energetics to community dynamics. $\mathrm{PhD}$ thesis. University of Amsterdam.

Thébault, E., and M. Loreau. 2005. Trophic interactions and the relationship between species diversity and ecosystem stability. American Naturalist 166:E95-E114.

van Leeuwen, A., A. M. de Roos, and L. Persson. 2008. How cod shapes its world. Journal of Sea Research 60:89-104. 
Vasseur, D. A. 2007. Populations embedded in trophic communities respond differently to coloured environmental noise. Theoretical Population Biology 72:186-196.

Vasseur, D. A., and J. W. Fox. 2007. Environmental fluctuations can stabilize food web dynamics by increasing synchrony. Ecology Letters 10:1066-1074.

Willson, M. F., and J. N. Womble. 2006. Vertebrate exploitation of pulsed marine prey: a review and the example of spawning herring Reviews in Fish Biology and Fisheries 16:183-200.
Yang, L. H., K. F. Edwards, J. E. Byrnes, J. L. Bastow, A. N. Wright, and K. O. Spence. 2010. A meta-analysis of resource pulse-consumer interactions. Ecological Monographs 80:125-151.

Yodzis, P., and S. Innes. 1992. Body size and consumer-resource dynamics. American Naturalist 139:1151-1175.

Associate Editor: Vlastimil Křivan Editor: Alice A. Winn

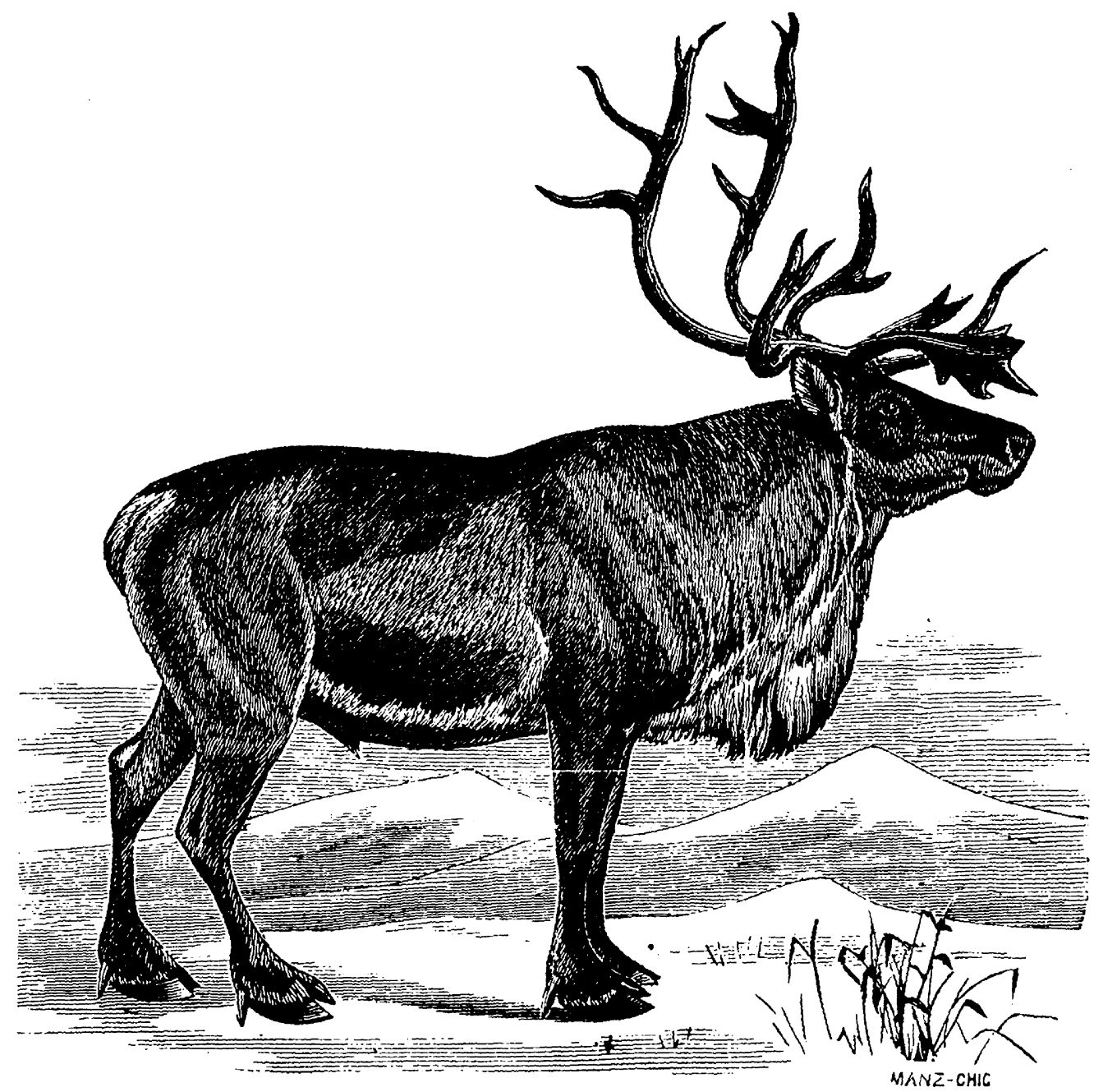

"Wild European reindeer-male." From the review of Caton's Summer in Norway (The American Naturalist, 1876, 10:39-42). 